\title{
Modelling and Numerical Simulations of In-Air Reverberation Images for Fault Detection in Medical Ultrasonic Transducers: A Feasibility Study
}

\author{
W. Kochański, ${ }^{1}$ M. Boeff, ${ }^{2}$ Z. Hashemiyan, ${ }^{1}$ W. J. Staszewski, ${ }^{1}$ and P. K. Verma ${ }^{3}$ \\ ${ }^{1}$ Department of Robotics and Mechatronics, AGH University of Science and Technology, Al. Mickiewicza 30, 30-059 Krakow, Poland \\ ${ }^{2}$ Department of Mechanical Engineering, Sheffield University, Sheffield S1 3JD, UK \\ ${ }^{3}$ Department of Medical Physics, Sheffield Teaching Hospital NHS Foundation Trust, Royal Hallamshire Hospital, \\ Glossop Road, Sheffield S10 2JP, UK
}

Correspondence should be addressed to W. J. Staszewski; w.j.staszewski@agh.edu.pl

Received 7 November 2014; Revised 26 January 2015; Accepted 17 February 2015

Academic Editor: Xinyong Dong

Copyright (C) 2015 W. Kochański et al. This is an open access article distributed under the Creative Commons Attribution License, which permits unrestricted use, distribution, and reproduction in any medium, provided the original work is properly cited.

\begin{abstract}
A simplified two-dimensional finite element model which simulates the in-air reverberation image produced by medical ultrasonic transducers has been developed. The model simulates a linear array consisting of 128 PZT-5A crystals, a tungsten-epoxy backing layer, an Araldite matching layer, and a Perspex lens layer. The thickness of the crystal layer is chosen to simulate pulses centered at $4 \mathrm{MHz}$. The model is used to investigate whether changes in the electromechanical properties of the individual transducer layers (backing layer, crystal layer, matching layer, and lens layer) have an effect on the simulated in-air reverberation image generated. Changes in the electromechanical properties are designed to simulate typical medical transducer faults such as crystal drop-out, lens delamination, and deterioration in piezoelectric efficiency. The simulations demonstrate that fault-related changes in transducer behaviour can be observed in the simulated in-air reverberation image pattern. This exploratory approach may help to provide insight into deterioration in transducer performance and help with early detection of faults.
\end{abstract}

\section{Introduction}

The performance of diagnostic medical ultrasound transducers can deteriorate during their working lifetime. This change in imaging performance is attributed to a number of factors which includes delamination between transducer layers, breakages in cables or components, short circuits, and weak or dead crystals $[1,2]$. There are number of quality assurance methods used for routinely checking transducer image quality. These methods include phantom based measurements and electrical methods.

Phantom based methods directly assess the imaging performance of a transducer by investigating aspects of image quality. For example the cyst detectability, axial, lateral or out of plane resolution, contrast sensitivity, imaging depth (through the low contrast penetration depth), and the resolution integral [3] can all be measured. Although phantom based measurements have the potential to provide performance indicators that are close to the clinical situation they do suffer from drawbacks and some studies have questioned their effectiveness [4]. The drawbacks of phantom based measurements include the inability of tissue equivalent phantoms to accurately match the acoustic properties (speed of sound, frequency dependent attenuation, and nonlinearity parameter) of human tissue. This has become more of an issue with the advent of broadband transducers so that now the acoustic properties of phantoms should ideally mimic that of human tissue over a wide frequency range. The acoustic properties of some gelatine based tissue equivalent phantoms can change over time and this can introduce an extra uncertainty in the interpretation of phantom based quality assurance measurements. Furthermore tissue equivalent phantom based measurements are generally performed using manual scanning techniques and often a subjective visual assessment of image quality is made.

Electrical measurements include the Sonora FirstCall probe tester. With this method the transducer is operated in 
a water environment, with transmitted pulses sent from the transducer reflected back to the transducer, via a metal plate, for detection. Proprietary transmitting/receiving electronics allow the sensitivity of individual crystals to be measured, alongside measurements of pulse width, pulse frequency content, and cable capacitance. The Sonora probe tester system however is expensive and relies on the availability of transmit-receive modules for different transducer models. Both phantom and electrical methods are time consuming and would require significant resources to perform routinely on, say, a weekly basis.

Traditionally national guidelines [5] suggest that quality assurance testing should be performed regularly, with different levels of testing at various repeat cycles. For example visual inspection for damage is recommended on a weekly basis whereas detailed image quality measurements are recommended typically twice a year. This means that a change in transducer performance may go unnoticed for a considerable length of time and this is a major drawback of such testing methodologies [2]. However more frequent regular testing (weekly or monthly) of image quality using either phantom or electrical methods is time consuming, would require significant capital and revenue resources, and would impact on patient waiting lists.

An alternative method [6] has been suggested to assess the imaging performance of transducers. This method relies on the assessment of the in-air reverberation pattern generated by a transducer. The method is a variation on an older method using a Perspex block [7] but is simpler to implement. Both methods are used to assess changes in the relative sensitivity (the ability to detect weak echoes from background noise) of the transducer. An in-air reverberation pattern is produced when a clean and dry transducer is operating in air. Acoustic pulses travel from the piezoelectric materials of the transducer and travel through the matching layers, lens layers, and protective layers of the transducer. The large impedance mismatch between the transducer and the air ensures there is no transmission of the sound beam into the air. The pulses are partially reflected back from each layer and are detected as echoes back at the piezoelectric crystals. A typical pulse can undergo a number of round trips through the layers before its amplitude is diminished due to attenuation and dispersion. Returning echoes are processed and displayed on the scanner monitor in the same way echoes from a clinical scan would be displayed.

The in-air reverberation pattern produced should depend on factors that broadly reflect the transmission and reflection properties of the transducer materials. Quality assurance guidelines recommend measuring the distance to the last visible reverberation plane [8] and using this as an indicator of transducer sensitivity. More recently work [9] has demonstrated that analyzing the full reverberation image may provide more accurate information on the performance of the transducer over its whole imaging plane. There is however very little understanding of relationship between transducer defects and changes in in-air reverberation pattern. Better understanding of the in-air reverberation pattern could lead to earlier detection of transducer faults and also to their cause. In order to better understand the in-air reverberation pattern that is generated, a linear array transducer model has been developed and numerical simulations of the in-air reverberation image have been investigated.

Various methods can be used for ultrasonic transducer modelling. Previous studies in this field include: mechanical and equivalent electrical circuit models (e.g., Mason model [10, 11], Redwood model [12], KLM model [11, 13], and Spice/PSpice models [14, 15]), transfer matrix method [16], distributed point source method [17], finite integration technique [18], Gaussian beam superposition [19], finite element analysis [20-24], acoustic radiation methods [25, 26], and various hybrid approaches of the above techniques [2729]. A comparative study of analytical, semianalytical, and numerical techniques is given in [30]. Various aspects related to coupled oscillator and finite element models of medical transducers are discussed in [26]. These two approaches are the most widely used methods for medical ultrasonic transducer modelling.

The paper aims to model pulse propagation through transducer layers using finite element (FE) analysis. It is anticipated that changes (or defects) in these layers will affect the transmission and reception properties of the transducer and therefore impact on the image quality of clinical images. The objective of this exploratory work is to determine how changes in the transmission and reflection properties of the various transducer layers can affect the whole reverberation pattern image.

The structure of the paper is as follows. Sections 2 and 3 briefly describe a typical medical ultrasonic transducer and the in-air reverberation test used for image quality assessment, respectively. A finite element model of the medical transducer is presented in Section 4. Numerical implementation of the transducer model and simple transducer defects are described in Section 5. Numerical results for defected single-crystal and multicrystal transducers are presented in Section 6. The focus is on qualitative analysis of the simulated in-air reverberation patterns. Finally, the paper is concluded in Section 7.

\section{Medical Ultrasonic Transducer}

Modern medical ultrasonic transducers are based on an array design with multiple small piezoelectric crystals (ceramic) as illustrated in Figure 1. The numbers of crystals in these arrays are often a multiple of sixty-four. There are generally three other material layers attached to each ceramics. These are a matching layer, a backing layer, and a lens layer. The four-layer structure (backing, ceramic, matching, and lens) is called a "finger" in this paper and is illustrated in Figure 1(b). A 128 ceramic transducer therefore consists of 128 fingers. The piezoelectric ceramic layer is used to generate and sense ultrasonic waves. An alternating electric current applied to the electrodes causes the piezoelectric ceramic to vibrate producing mechanical waves that propagate through the various layers of the transducer. Reflected mechanical waves come back to the piezoelectric layer and produce an electrical current that, after a number of processing steps, is used to generate an ultrasonic image. Piezoceramics or 


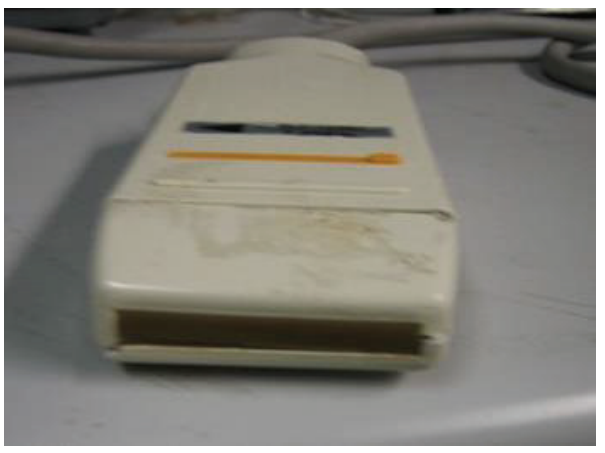

(a)

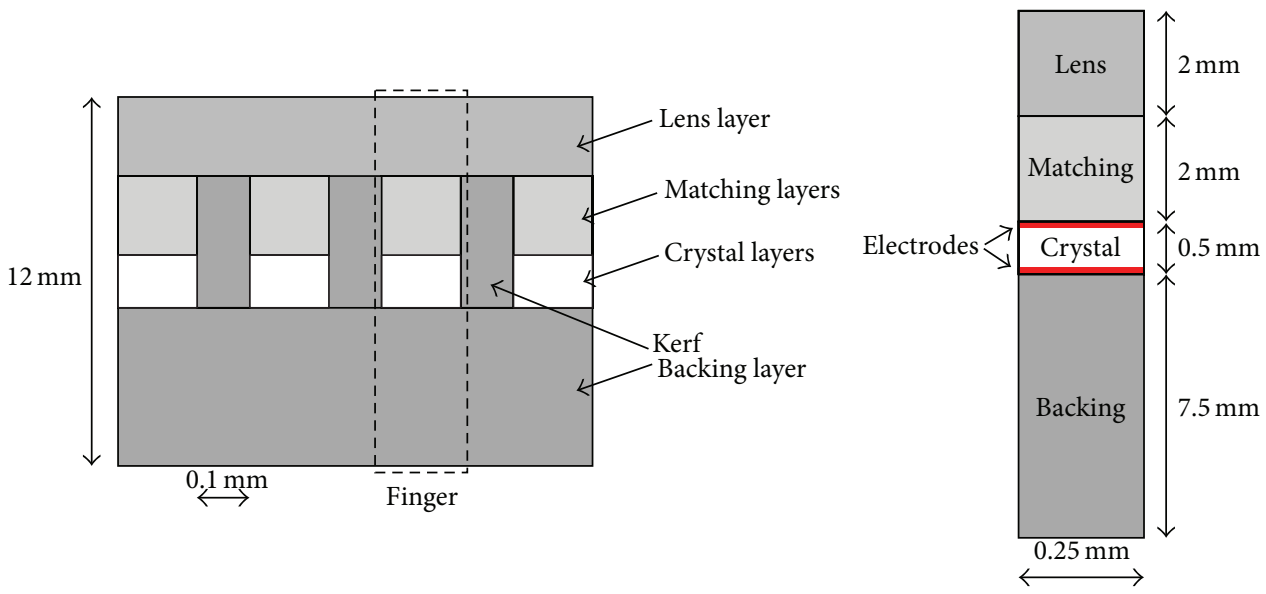

(b)

Figure 1: Example of medical ultrasonic transducer: (a) general view; (b) internal design-general view (left); geometry of one element (right).

piezocomposites are common materials that are used as piezoelectric crystals.

A matching layer is required to improve impedance mismatch between the piezoelectric crystals and human skin. In practice the matching layer consists of a number of thin layers. The impedance of these layers gradually changes from the impedance of the piezoelectric crystal to the impedance of the human skin. A backing layer is used to attenuate vibration of the ceramic in the direction opposite to ultrasonic wave transmission and to prevent unwanted acoustic reverberations between the matching layer and the backing material. This layer is usually manufactured from a heavy, metal-based material. Usually a backing with a high ultrasonic attenuation is selected to damp ultrasonic waves. Often the backing layer has similar acoustic impedance as the ceramic layer to avoid possible reflections. An acoustic lens is often used to focus the ultrasound beam in a plane which is perpendicular to the imaging plane. The lens layer usually consists of a material with acoustic impedance that is between the matching layer and human skin. The gap between crystals $(0.1 \mathrm{~mm})$ is called kerf, the kerfs often filled with a damping material having low acoustic impedance that blocks and absorbs the transmission of vibration between adjoining elements, or they may be air filled. The height of all four layers can typically be approximately $12 \mathrm{~mm}$. The thickness of the ceramic layer is defined by the required frequency of the transducer. Since a thin piezoceramic wafer vibrates with a wavelength that is twice its thickness $\lambda=2 d$. Therefore piezoelectric crystals are made with a thickness of around $1 \backslash 2$ of the demanded wavelength, but when piezoelectric crystals are inserted between the matching layer and the backing, this frequency decreases and consequently, the thickness must be adapted.

\section{In-Air Reverberation Test}

It is well known that when ultrasonic transducers are extensively used for a long period of time image quality deteriorates [1-3]. The in-air reverberation test is a method for assessing changes in medical ultrasound transducer sensitivity. A transducer with a dry and clean surface is operated in air. It is important to ensure that the surface of the transducer is dry and clean because the test relies on there being a large impedance mismatch between the transducer surface layer and air. Furthermore this impedance mismatch should not vary between measurements, unless it is due to intrinsic changes in transducer properties. Ultrasonic waves generated in ceramic layers travel through the remaining layers of the transducer and reach the air boundary. When the transducer is not in contact with another object, the difference in acoustic impedance between the lens layer (this is often the surface layer) and the air is significantly large. As a result, ultrasonic 


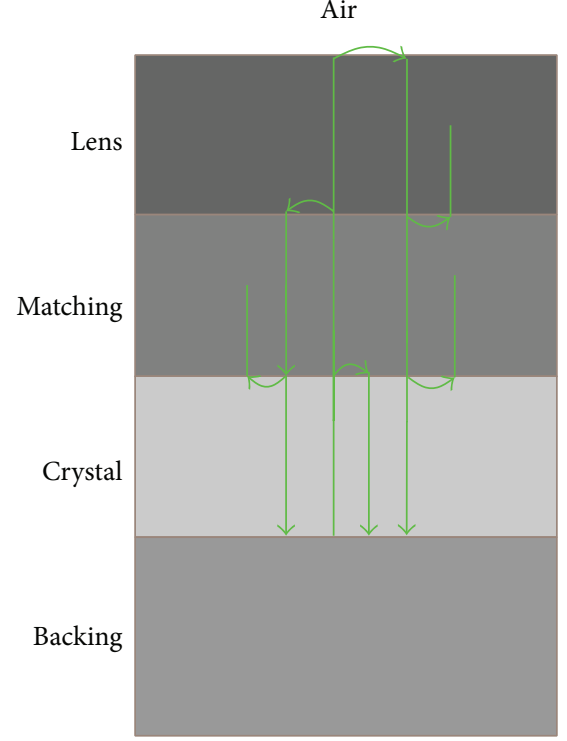

(a)

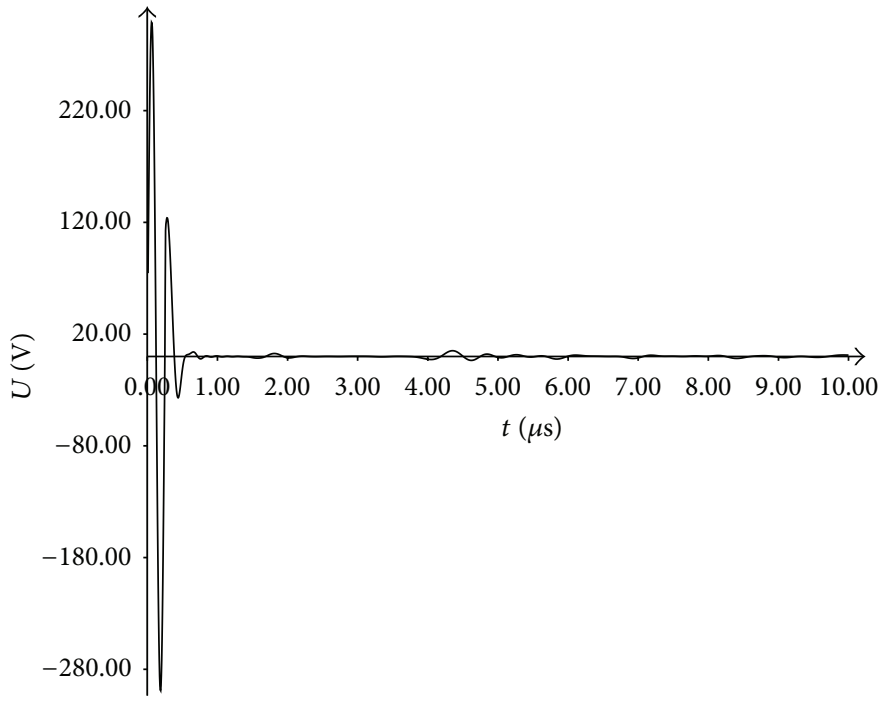

(b)

FIGURE 2: Graphical illustration of the in-air reverberation test: (a) ultrasonic waves travelling in the transducer: (b) example of resulting voltage signal.

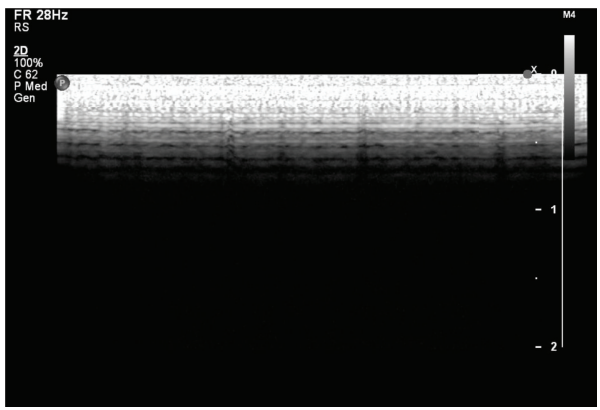

(a)

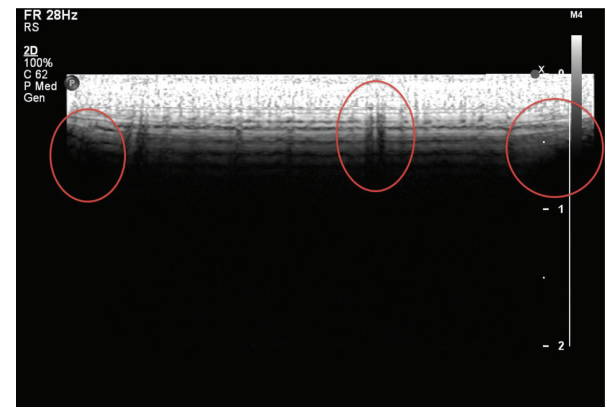

(b)

FIGURE 3: Ultrasonic images from a brand new transducer (a): the same transducer after 20 months of operation (b). Image disturbances are encircled.

waves are reflected and travel back through all layers of the transducer.

It is clear that waves travelling through all these layers are additionally reflected from the boundaries between the layers, as illustrated in Figure 2(a). The waves bounce forward and backward producing voltage signals, at the ceramic layer, that resemble the characteristic signal shown in Figure 2(b). The scanner processes the voltage signals arriving at the piezoelectric ceramic in a transducer array and produces an image which is the in-air reverberation pattern. Figure 3 gives examples of reverberation patterns from a new transducer (left hand side) and the same transducer after 20 months of operation (right hand side). There are noticeable differences in the reverberation pattern. The right hand image shows that the depth of the reverberation pattern at both ends of the transducer has reduced; the implication is that the transducer sensitivity has diminished in these regions. There are also dark axial bands running down the length of the central region of the transducer which may be due to localized changes in the electromechanical properties of the piezoelectric, matching, or lens layers. These changes in the reverberation pattern may be indicative of impaired transducer performance which in turn may have implications for diagnostic accuracy.

\section{Wave Propagation in Ultrasonic Transducers: Theoretical Background}

An ultrasonic wave is an elastic deformation propagating through a material. When a cube of solid is excited by an external force this deformation can be quantified using strains. The relationship between strains $\mathbf{S}$ and stresses $\mathbf{T}$ can be expressed using the generalised form of Hooke's law

$$
\mathbf{S}=\delta \mathbf{T}
$$


where $\delta$ is compliance, that is, the inverse of stiffness. The motion of a solid can be described using Newton's law as

$$
\rho \ddot{\mathbf{u}}=\nabla \cdot \mathbf{T},
$$

where $\mathbf{u}$ is mechanical displacement and $\rho$ is density. Then, combining Hooke's and Newton's laws will lead to the classical wave equation that can be given in the following form:

$$
c^{2} \nabla^{2} \mathbf{u}=\mathbf{u}_{t t}
$$

where the constant $c$ comes from mass density and elasticity and represents wave velocity. Both longitudinal and shear waves can propagate in a solid cube. The longitudinal and shear wave velocities are equal to

$$
c_{s}=\sqrt{\frac{\widehat{E}}{2 \rho(1+\nu)}}, \quad c_{l}=\sqrt{\frac{\widehat{E}(1-\nu)}{\rho\left(1-\nu-2 \nu^{2}\right)}},
$$

respectively, where $\widehat{E}$ is Young's modulus and $v$ is Poisson's ratio.

Ultrasonic waves, that propagate in various layers of the transducer, are generated by piezoelectric ceramic. These crystals convert electrical energy into mechanical energy and vice versa. The direct piezoelectric effect is the ability of a material to generate an electrical charge in proportion of an applied force. The inverse piezoelectric effect is the ability of a material to deform under an applied electrical field. An electric potential loaded between top and bottom surfaces of the piezoelectric ceramic results in deformation of the crystal. Both piezoelectric effects are used in medical ultrasonic transducers to generate and sense ultrasonic waves.

The electric behavior of the material can be described as

$$
\mathbf{D}=\varepsilon \mathbf{E},
$$

where $\mathbf{D}$ is the electric displacement, $\varepsilon$ is permittivity, and $\mathbf{E}$ is electric field strength. This equation can be combined with Hook's law and rearranged to give

$$
\begin{aligned}
& \mathbf{D}=\mathbf{d} \mathbf{T}+\varepsilon^{T} \mathbf{E}, \\
& \mathbf{S}=\varepsilon^{E} \mathbf{T}+\mathbf{d}^{t} \mathbf{E},
\end{aligned}
$$

where the superscript $t$ indicates the transpose and the superscripts $T$ and $E$ represent constant stress and electric fields, respectively. Thus $\varepsilon^{T}$ is the permittivity for constant mechanical stress and $\delta^{E}$ is the compliance for a constant electrical field. These two well-known coupled constitutive linear equations govern the energy transfer; that is, (6) and (7) represent the direct and inverse piezoelectric effects, respectively. At this point it is important to mention that the strain and stress rank two tensors in the above equations can be rearranged to vectors with 6 components due to their symmetry. This is why the compliance $\delta$ normally a rank four tensor appears to be a $6 \times 6$ matrix. This notation, often used when finite element analysis is performed, is called the Voigt notation. It is important to note that (6) and (7) contain a large number of variables. However, in practice the number of these variables is significantly reduced due to the symmetry of the problem investigated.

Equation (7) can be simplified since there are two effects of main interest in the region of actuating piezoelectric crystals. For the piezoelectric transversal case, where the mechanical stress works orthogonal to the electrical field, the following can be obtained:

$$
\mathrm{S}_{1}=\delta_{11}^{\mathrm{E}} \cdot \mathrm{T}_{1}+\mathrm{d}_{31} \cdot \mathrm{E}_{3},
$$

whereas, for the piezoelectric longitudinal effect where the mechanical stress is parallel to the electrical field, (7) can be rewritten as

$$
S_{3}=\delta_{33}^{E} \cdot T_{3}+d_{33} \cdot E_{3} .
$$

The equation of electric balance is needed additionally to solve the problem; that is,

$$
\nabla \cdot \mathbf{D}=0 .
$$

It is clear that the strain and electric matrices can be expressed in terms of mechanical displacements and voltage $\varphi$ as

$$
\mathbf{S}=\nabla^{S} \mathbf{u}, \quad \mathbf{E}=-\nabla \varphi .
$$

The entire problem needs to be complemented by the appropriate boundary conditions. Prescribed voltages are used in the current analysis to excite the piezoelectric elements.

4.1. Reflection and Attenuation. When ultrasonic waves propagate in the transducer only part of the wave is transmitted through various boundaries, as illustrated in Figure 2. The remaining part is reflected and dissipated. Reflection and dissipation depend on material properties. The reflection can be described using the reflection coefficient defined as

$$
R=\left(\frac{Z_{2}-Z_{1}}{Z_{2}+Z_{1}}\right)^{2}
$$

where $Z$ is the acoustic impedance of the material. The impedance can be calculated as

$$
Z=\rho \cdot c .
$$

With an increasing difference between $Z_{1}$ and $Z_{2}$ the reflection coefficient $R$ goes up and this leads to a stronger reflection. When $Z_{1}$ and $Z_{2}$ have the same value, the reflection coefficient is zero and no reflection takes place.

Once the wave travels through various layers of material the energy is partially lost due to spreading, scattering, and absorption. Absorption stands for the conversion of kinetic energy to other forms of energy. Scattering means the reflection of sound directions other than the original direction of wave propagation. Both effects together account for the attenuation of ultrasonic waves. A change in the waves amplitude can be captured with Beer Lambert's law which gives the intensity of an ultrasonic wave after the wave has travelled through a layer of material with thickness $z$ as

$$
I(z)=I_{0} e^{-\alpha z},
$$

where $I_{0}$ is the amplitude the wave at $z=0, \alpha$ is the attenuation coefficient, and $I(z)$ is the amplitude at the point $z$. 
TABLE 1: Dimensions for the one-element transducer.

\begin{tabular}{lcccc}
\hline Name & Backing layer & Crystal & Matching layer & Lens \\
\hline Width & $0.25 \mathrm{~mm}$ & $0.25 \mathrm{~mm}$ & $0.25 \mathrm{~mm}$ & $0.25 \mathrm{~mm}$ \\
Thickness & $7.5 \mathrm{~mm}$ & $0.5 \mathrm{~mm}$ & $2 \mathrm{~mm}$ & $2 \mathrm{~mm}$ \\
\hline
\end{tabular}

\section{Finite Element Model of the Defected Medical Ultrasonic Transducer}

Finite element analysis, based on ANSYS [31], was used to simulate wave propagation in a medical ultrasonic transducer. The entire derivation process of integral forms of partial differential equations is not presented here and interested readers are referred to $[26,32]$. The physical problem described in Section 4 can be solved through approximations of the relevant partial differential equations and discretization of the transducer geometry. Once the geometry is discretized into small finite elements the solution is obtained for nodes of these elements. The required solutions for arbitrary positions are obtained using a linear combination of polynomial interpolation functions, as described in $[26,32]$. The entire analysis is performed in the time domain using a step-bystep time integration scheme to obtain displacements and voltages. The latter is used to compute reverberation patterns. This section describes details related to the geometry, material parameters, and excitation.

5.1. Spatial and Temporal Discretization. A simple 2D model of the medical ultrasonic transducer, presented in Figure 1, was used in numerical simulations. The study involved two different transducer configurations. Firstly, a one element transducer was used to investigate the effect of defect severity for various defected layers of transducer. Then 128-finger transducer was simulated for different types of defects by using coupled-field quadrilateral solid elements. The thickness of the ceramic layer was defined by the required frequency of the transducer as a thin wafer element vibrates with a wavelength that is twice its thickness $\lambda=2 d$. Therefore piezoelectric ceramics are usually made with a thickness of around $1 / 2$ of the demanded wavelength. The dimensions of the piezoceramic crystal and all layers involved are given in Table 1.

All material layers were discretized into $0.05 \times 0.05 \mathrm{~mm}$ elements. The one-finger transducer was modelled using 1200 coupled-field quadrilateral solid elements and 1464 nodes. The 128-finger transducer consisted of 168520 coupledfield quadrilateral solid elements and 180555 nodes. Large numbers of elements were selected to avoid nonphysical frequencies and direction dependent dispersive character, following recommendations given in [33]. The same degree of discretization was maintained throughout the mesh to avoid directionality and spurious internal reflections.

The choice of the right element type is a key point for appropriate finite element calculations. The PLANE13 elements were used to model the piezoelectric layer of the transducer. This element is defined by four nodes and 3 degrees of freedom per node (i.e., two displacements and voltage). The backing, matching, and lens layers were modelled using
TABLE 2: Piezoceramic material properties used in numerical simulations.

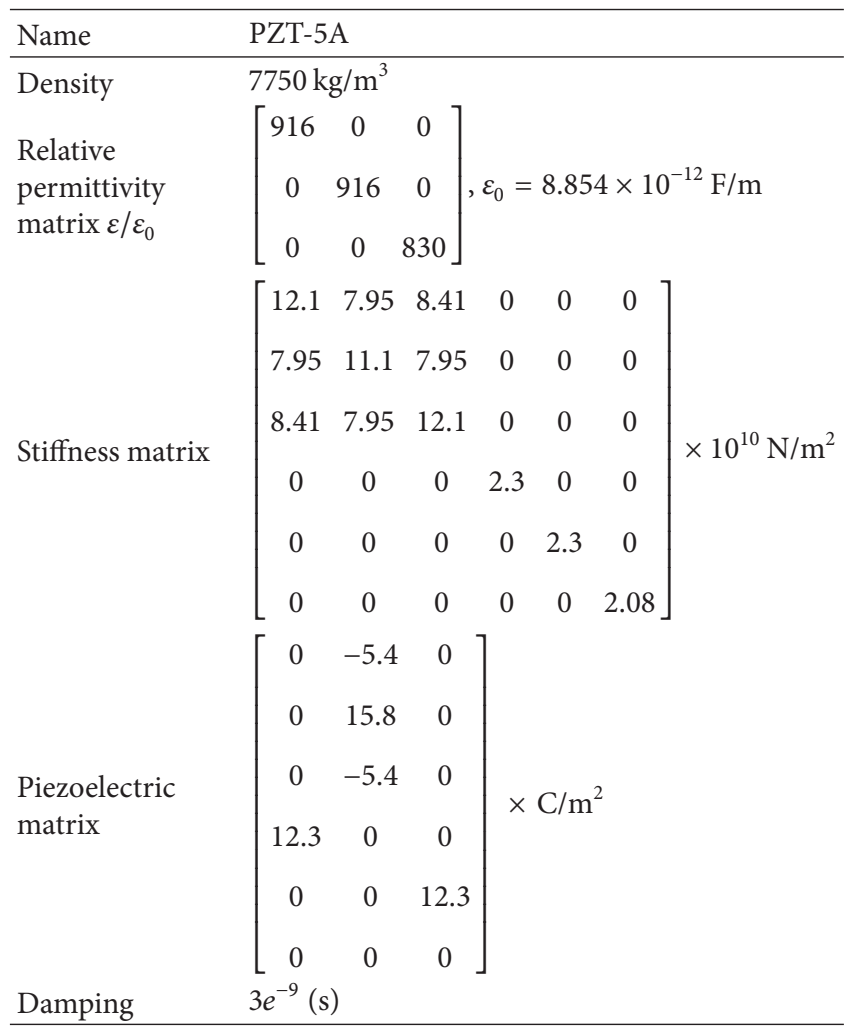

the PLANE42 elements defined by 4 nodes with 2 degrees of freedom at each node (i.e., two displacements). The time step was always selected to achieve the Courant-Friedrichs-Lewy (CFL) stability condition. For the 2D case the CFL condition states that

$$
C=\frac{v_{x} \Delta t}{\Delta x}+\frac{v_{y} \Delta t}{\Delta y} \leq C_{\max }
$$

where $C$ is the Courant number, $v_{x}, v_{y}$ are velocities, $\Delta x, \Delta y$ are length intervals, and $\Delta t$ is time step.

Time characteristics from transient analysis were integrated using two different ranges; that is, the time step equal to $1 e^{-8} \mathrm{~s}$ was used for the $0-5 \mu$ s interval and then the time step equal to $5 e^{-8} \mathrm{~s}$ was applied for the $5-10 \mu \mathrm{s}$ interval. Smaller intervals were used in the first time range due to the fact that the whole range of arriving to the ceramic has to be covered.

5.2. Material Properties. A PZT-5A piezoceramic material was used to model the piezoelectric layer [34-36]. The thickness of the piezoceramic layer is related to the frequency of the wave and equals half of the wavelength. Table 2 gives 
TABLE 3: Materials properties of backing, matching, and lens layers used in numerical simulations.

\begin{tabular}{lccc}
\hline Name & Backing layer & Matching layer & Lens layer \\
\hline Young's modulus & $8.02 e 10 \mathrm{~Pa}$ & $9.345 e 9 \mathrm{~Pa}$ & $0.31 e 10 \mathrm{~Pa}$ \\
Poisson's ratio & 0.3 & 0.34 & 0.3 \\
Density & $11000 \mathrm{~kg} / \mathrm{m}^{3}$ & $1340 \mathrm{~kg} / \mathrm{m}^{3}$ & $1200 \mathrm{~kg} / \mathrm{m}^{3}$ \\
Damping & $1.5 e-8$ & $6 e-9$ & $10 e-10$ \\
\hline
\end{tabular}

TABLE 4: Defect simulations: values of reduced Young's modulus for different transducer layers.

\begin{tabular}{lcccc}
\hline $\begin{array}{l}\text { Damage severity } \\
\text { (or damage case) }\end{array}$ & $\begin{array}{c}\text { Backing layer damage } \\
\text { Young's modulus [Pa] }\end{array}$ & $\begin{array}{c}\text { Matching layer damage } \\
\text { Young's modulus [Pa] }\end{array}$ & $\begin{array}{c}\text { Lens layer damage } \\
\text { Young's modulus [Pa] }\end{array}$ & $\begin{array}{c}\text { Crystal layer damage } \\
\text { Stiffness denominator }\end{array}$ \\
\hline 1 & $1 \cdot 10$ & $1 \cdot 10$ & $1 \cdot 10^{3}$ & $1 \cdot 10^{5}$ \\
2 & $1 \cdot 10^{3}$ & $1 \cdot 10^{3}$ & $1 \cdot 10^{7}$ & 1000 \\
3 & $1 \cdot 10^{5}$ & $1 \cdot 10^{5}$ & $1 \cdot 10^{8}$ & 4 \\
4 & $1 \cdot 10^{7}$ & $1 \cdot 10^{7}$ & $5 \cdot 10^{8}$ & 2 \\
5 & $1 \cdot 10^{9}$ & $1 \cdot 10^{9}$ & $1 \cdot 10^{9}$ & 1.7 \\
6 & $3 \cdot 10^{9}$ & $3 \cdot 10^{9}$ & $2 \cdot 10^{9}$ & 1.4 \\
7 & $6 \cdot 10^{9}$ & $6 \cdot 10^{9}$ & $3 \cdot 10^{9}$ & 1.2 \\
\hline
\end{tabular}

a summary of electromechanical properties selected for the piezoceramic crystal.

Backing layers are usually selected from a material that meets two conditions. Firstly the ability to attenuate sound waves should be relatively high and secondly the acoustic impedance should be similar to the acoustic impedance of the piezoceramic layer to avoid wave reflections from the piezoceramic-backing layer boundary due to impedance mismatch. Therefore a Tungsten-Epoxy material was used to model the backing layer. The matching layer was modeled using Araldite material. The lens layer was modeled using the properties of Perspex material. Mechanical properties of the matching, backing, and lens layers are given in Table 3. All material properties were taken following previous investigations reported in [37].

5.3. Excitation. A one cycle sine wave pulse of $4 \mathrm{MHz}$ frequency and $300 \mathrm{~V}$ amplitude was used as the input signal to the upper surface of the piezoceramic crystal; a constant zero voltage was applied to the opposite surface of the crystal. When receiving signals were gathered, the upper electrode was grounded, and the voltage signal was collected from the lower electrode. Input voltage signals were applied to subsequent crystals with the time delay of $0.015 \mu \mathrm{s}$ in the 128 element model. The delays were applied from the centre to the sides of the transducer.

5.4. Defect Modelling. As material faults are often connected with stiffness reduction, transducer defects were simulated using the reduction of stiffness to various transducer layers. The largest reduction (i.e., the smallest value of stiffness) used in the analysis was related to the full breakage of the involved element. Defects in all transducer layers were simulated. Stiffness reduction was maintained through Young's modulus. Young's modulus was reduced for the matching, backing, and lens layers. The coefficients of stiffness matrix were reduced for the piezoelectric layer through the stiffness denominator. Defects were simulated at different locations using eight various severity levels, where damage severity case 1 corresponds to full breakage of the relevant layer. Table 4 gives information about all defects in various transducer layers. The parameter investigated is reduced by the value given in the last column in Table 4.

\section{Numerical Simulation Results}

This section presents numerical results from the finite element analysis of a defected medical ultrasonic transducer. Firstly, initial results are presented to investigate wave propagation reflections from various boundaries. Then reverberation patterns are investigated for the one-element transducer and various damage severities. Finally, selected defects are simulated in the 128-finger transducer and the relevant reverberation patterns are analyzed.

6.1. Initial Results. A one-element transducer was modelled initially to investigate wave propagation. The excitation signal was applied to the transducer, as explained in Section 5.3. The response signal, captured at the bottom of the piezoceramic element, is shown in Figure 4. The first part of the signal (from 0 to $0.25 \mu \mathrm{s}$ ) is the original $300 \mathrm{~V}$ input pulse. Then the original impulse travels through the transducer towards the backing and matching layers. The wave propagation paths in the transducer are illustrated in Figure 4(a). The signal travelling downwards travels through the backing layer and is attenuated quickly due to the large value of damping of this layer. The signal travelling upwards travels through the ceramic layer, matching layer, and lens layer until it arrives at the top of the transducer, where the full reflection takes place due to the large value of reflection coefficient between the lens and air layers. This signal is also attenuated but the loss of energy is smaller than for the signal 


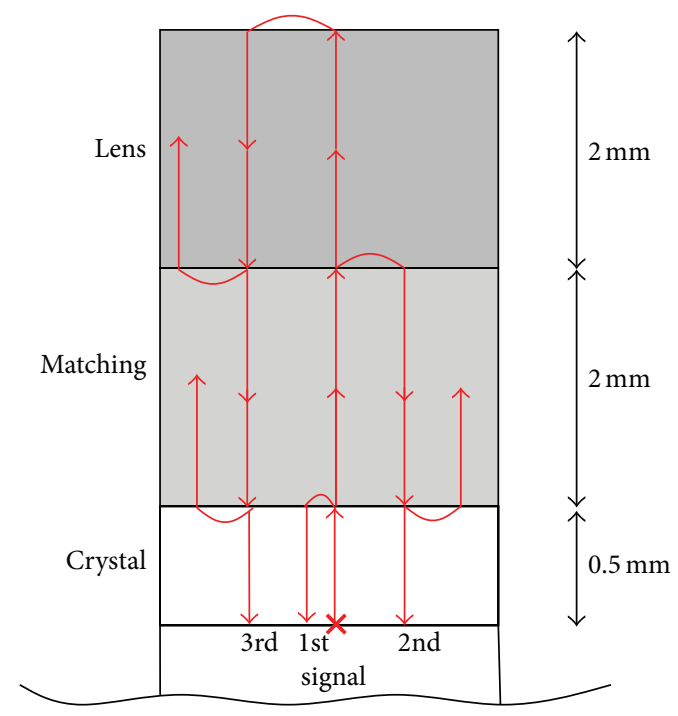

(a)

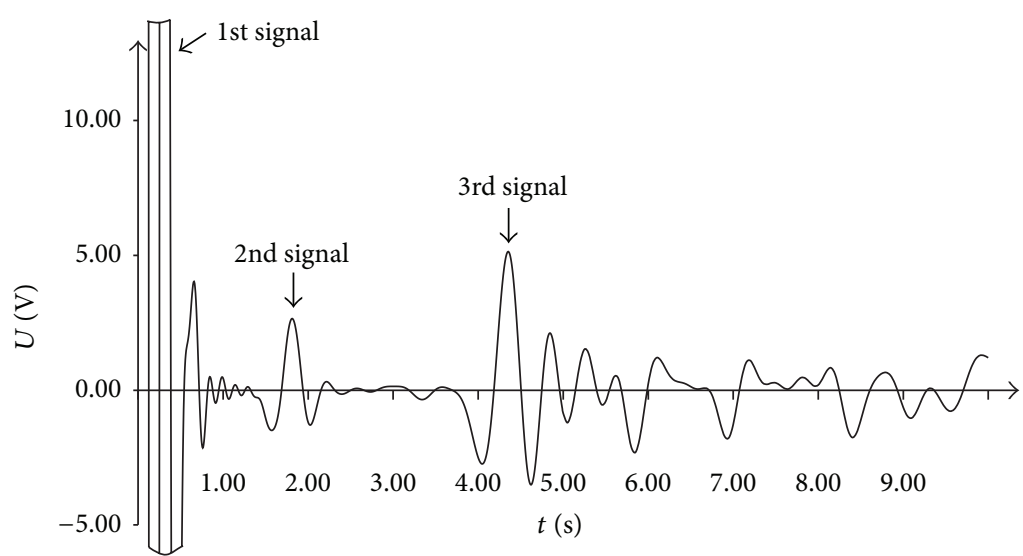

(b)

FIGURE 4: Ultrasonic wave propagated through the transducer: (a) illustration of analysed wave propagation paths 1, 2, and 3; (b) simulated response signal with indicated reflections for the analysed propagation paths $(1,2$, and 3$)$.

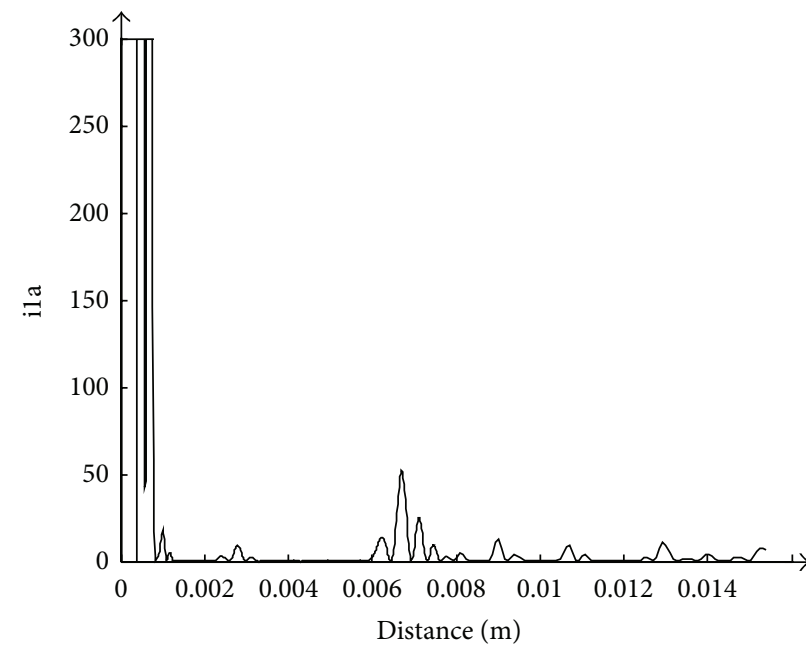

(a)

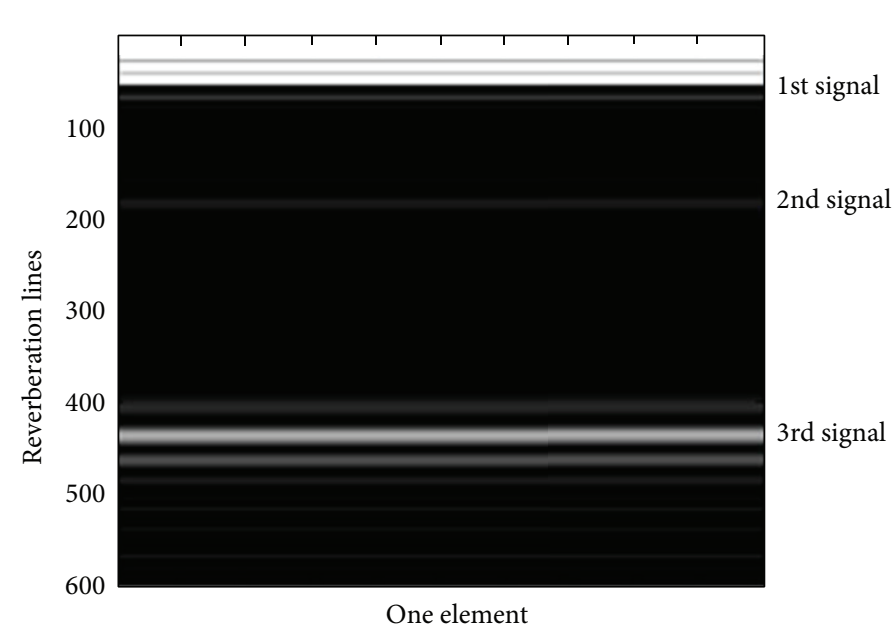

(b)

Figure 5: Voltage amplitude intensity (a) and the corresponding in-air reverberation pattern (b) for the one element medical ultrasonic transducer. Signals 1, 2, and 3 on the right hand side correspond to the signal paths and reflections indicated in Figure 4.

travelling downwards. The upwards signal traveling through the transducer also gets reflected from various boundaries between layers. Three different paths, indicated as integer numbers, are illustrated in Figure 4(a). These are reflections from (1) crystal and matching layer boundary; (2) matching layer and lens boundary; and (3) lens-air boundary. The actual reflections arriving at the bottom of the piezoceramic crystal are indicated in the response wave propagation signal shown in Figure 4(b). Theoretical arrival time values for the analyzed 1st, 2 nd, and 3 rd signals were calculated as, 0.22 , 1.52 , and $4 \mu \mathrm{s}$. Figure 4 (b) shows that the analytically calculated values are matched quite well by the simulated arrivals, given the model simplicity and dimensions [38].
Piezoceramic crystal voltage responses were converted into in-air reverberation image patterns using a standard image display method for medical ultrasonic transducers [39]. These patterns represent 8 bit images of amplitude intensities. Figure 5 gives an example of the voltage response signal and the corresponding in-air reverberation pattern for a one-element transducer. Bright regions in the image represent pulses arriving back at the ceramic, after reflections from the transducer layers, at various times after the initial excitation. The brightness in the image in Figure 5 is proportional to the amplitude of the voltage generated by the piezoelectric ceramic when the returning pulse excites the ceramic. The results are obtained 600 reverberation lines 
where $6 \mu$ s corresponded to 600 lines. A reverberation band at a depth of approximately 45 (corresponds to $0.45 \mu \mathrm{s}$ ) appears due to the partial reflection from the crystal-matching layer boundary. A reverberation band at a depth of approximately 180 (corresponds to $1.8 \mu \mathrm{s}$ ) appears due to the partial reflections at the matching-lens boundary. A further reverberation band at a depth of approximately 400 (corresponds to $4 \mu \mathrm{s}$ ) appears due to the total reflection at the lens-air border.

6.2. Reverberation Patterns for a One-Finger Transducer. The one-element ultrasonic transducer was further analysed to investigate the effect of defects in various transducer layers. The defect was simulated following the procedure described in Section 5.4. Eight damage cases, with the defect severities given in Table 4, were investigated. Figure 6 shows the numerical simulation results. Voltage response signals for various damage severities investigated are given on the left hand side of each figure, whereas the corresponding in-air reverberation patterns are presented on the right hand side of each figure. The reverberation patterns are given for eight different severities investigated, where 1 corresponds to the full breakage of the given layer and 8 represents the smallest damage severity investigated, as explained in Table 4.

The results show that when the backing layer is damaged less attenuation can be observed in voltage signals. Voltage amplitudes and numbers of oscillations increase with damage severities in Figure 6(a). As a result the amplitude intensity increases and this is reflected by white areas of in-air reverberation patterns. Significant change can be observed for the 5th damage case investigated corresponding approximately to $98.3 \%$ Young's modulus reduction (i.e., from the initial value of $8.02 e 10$ to the reduced value of $1.0 e 9 \mathrm{~Pa}$ ). Some extra intense white lines can be also observed at the bottom of in-air reverberation patterns afterwards, that is, for larger damage severities (i.e., damage cases 1-4).

When the matching layer is damaged clear difference can be observed when Young's modulus is reduced approximately by $67.9 \%$ (i.e., from the initial value of $9.345 e 9 \mathrm{~Pa}$ to the reduced value of $3.0 e 9 \mathrm{~Pa}$ ). This corresponds to the 6th damage case in Figure 6(b). Clear differences at the end of the voltage responses and the complete disappearance of the corresponding amplitude intensity lines in the in-air reverberation patterns can be observed in Figure 6(b).

The damaged crystal layer produces a significant difference in in-air reverberation patterns after approximately $25 \%$ stiffness reduction (i.e., the denominator of the stiffness matrix was equal to 4). As a result in-air reverberation patterns for the damage cases 1-4 display an increased number of amplitude intensity bright lines. This change is quite dramatic for the 1-3 damage cases investigated.

When the lens layer is damaged, significant differences can be observed after approximately $35 \%$ reduction of Young's modulus, that is, for the 7th damage case investigated. The change due to damage in the in-air reverberation patterns in Figure 6(d) corresponds to the disappearance of the lens-air reflections lines at the bottom and to the appearance of the extra intensity lines in the middle of the in-air reverberation patterns.
In summary, damage to crystal followed by the damage to lens has the most deteriorating effect on in-air reverberation pattern. The former was expected before the analysis has been performed. Damage to the backing layer is the most difficult one to detect for small reductions of Young's modulus. The results also show that the disappearance of the lens-air reflection lines in the in-air reverberation patterns could indicate damage to the matching or lens layers. However, the latter exhibit some extra lines in the middle of in-air reverberation images. Damage to the backing and crystal layers exhibited extra intensity lines in the in-air reverberation patterns. This effect is more pronounced (i.e., appears in the whole image) when the crystal layer is damaged.

6.3. Reverberation Patterns for a Multiple Finger Transducer. The 128-finger transducer was modelled to investigate whether it is possible to scale the damage-related effects observed in Section 6.2 for the one-finger transducer and also to see whether it is possible to localize damage, that is, to identify damaged fingers in the transducer. The in-air reverberation pattern for the 128-finger healthy transducer is presented in Figure 7.

The in-air reverberation pattern for the 128-finger transducer in Figure 7 exhibits amplitude intensity lines due to reflections (signals 1,2, and 3) in a similar manner the one element-transducer in Figure 5 as explained in Section 6.1. Additionally significant border effects, encircled in Figure 7, can be clearly observed due to the air boundary at the sides of the transducer. These effects, seen for the first and last 20 fingers of the transducer, are particularly pronounced in the left and right top corners of the in-air reverberation patterns.

The simulated results for the 128 -finger damaged transducer are given in Figure 8, as an example. The results show the most severe damage case 1 (see Table 4 for explanation) for four different transducer layers. The border effects, explained above, can be clearly observed in all images.

Figure 8(a) shows the ultrasonic in-air reverberation image for the broken backing layer. Young's modulus of the six middle fingers was reduced to $10 \mathrm{~Pa}$. When Figure 8(a) is compared with Figure 7, the damage is apparent immediately. White amplitude intensity lines can be seen in the middle top area of the image. This effect is very similar to the image for the one-element transducer in Figure 6(a).

Figure $8(\mathrm{~b})$ gives the in-air reverberation image for the transducer with broken matching layer at the 59th and 70th fingers. The electrodes at the broken fingers receive a strong signal for the first reverberation pattern at the depth of 50 (corresponds to $0.5 \mu \mathrm{s}$ ). Further signals are not received due to the high reflection coefficient at the matching-ceramic boundary. As a result gaps in the pattern (i.e., dark areas) can be seen. Similar behavior has been observed for the one-finger transducer in Figure 6(b). The fingers in the close neighborhood of the broken matching layer are also slightly affected.

The ultrasonic in-air reverberation image for a transducer with six broken piezoelectric crystals is shown in Figure $8(\mathrm{c})$. The damaged fingers are in the central region of the transducer array. A clear pattern due to damage can be observed in the middle part of the image. This effect 

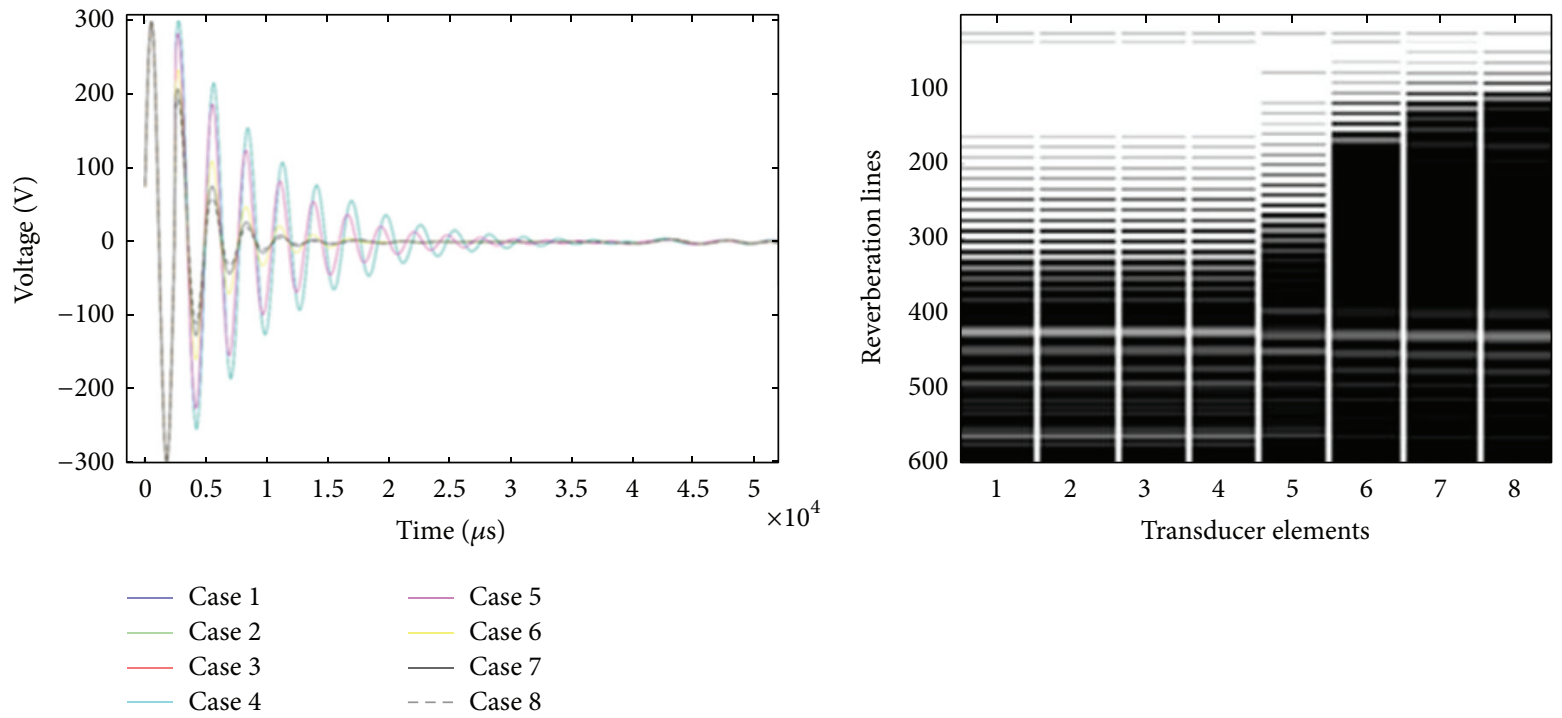

(a)
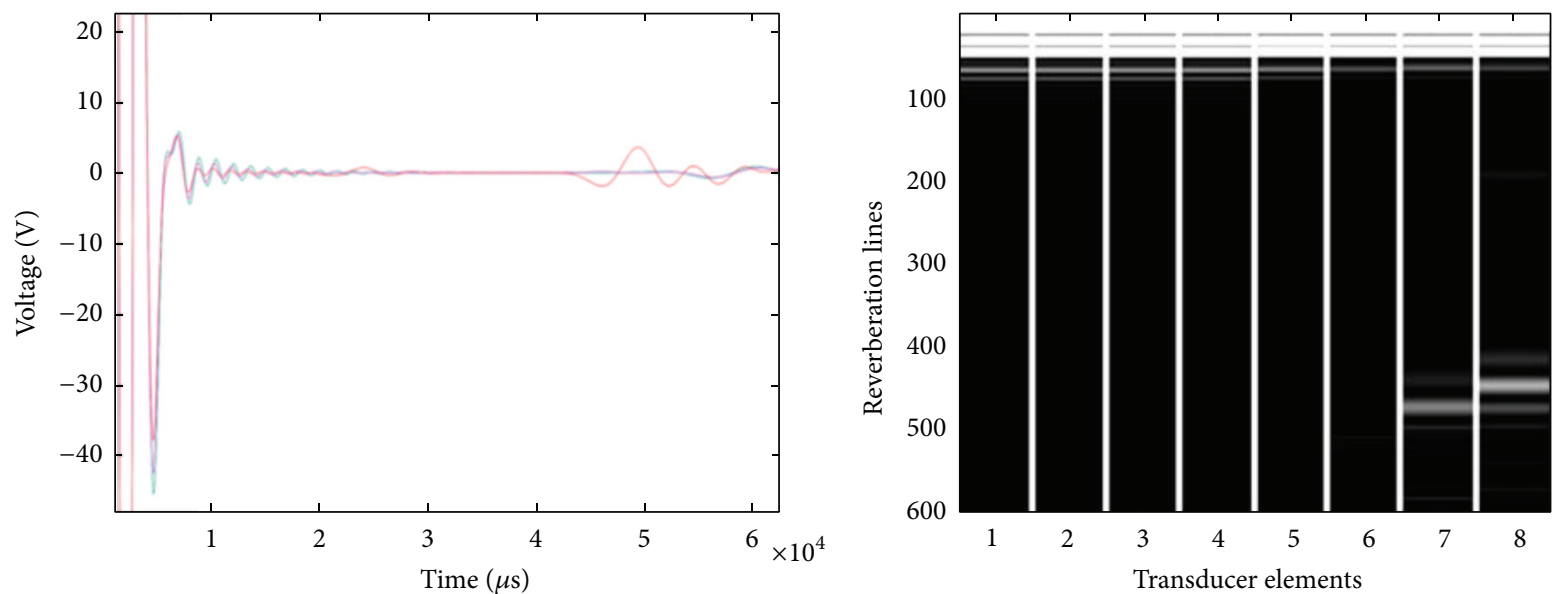

(b)
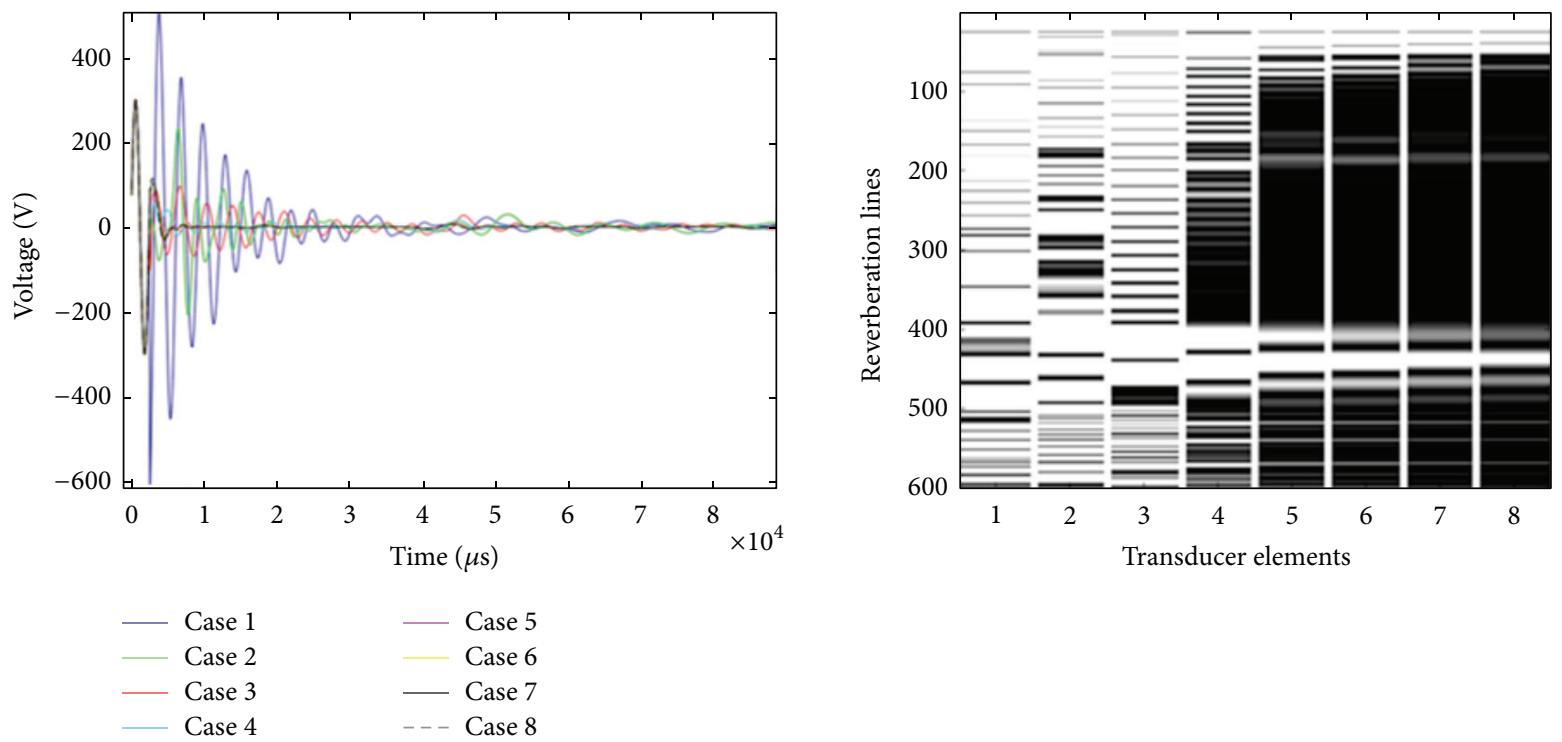

(c)

Figure 6: Continued. 

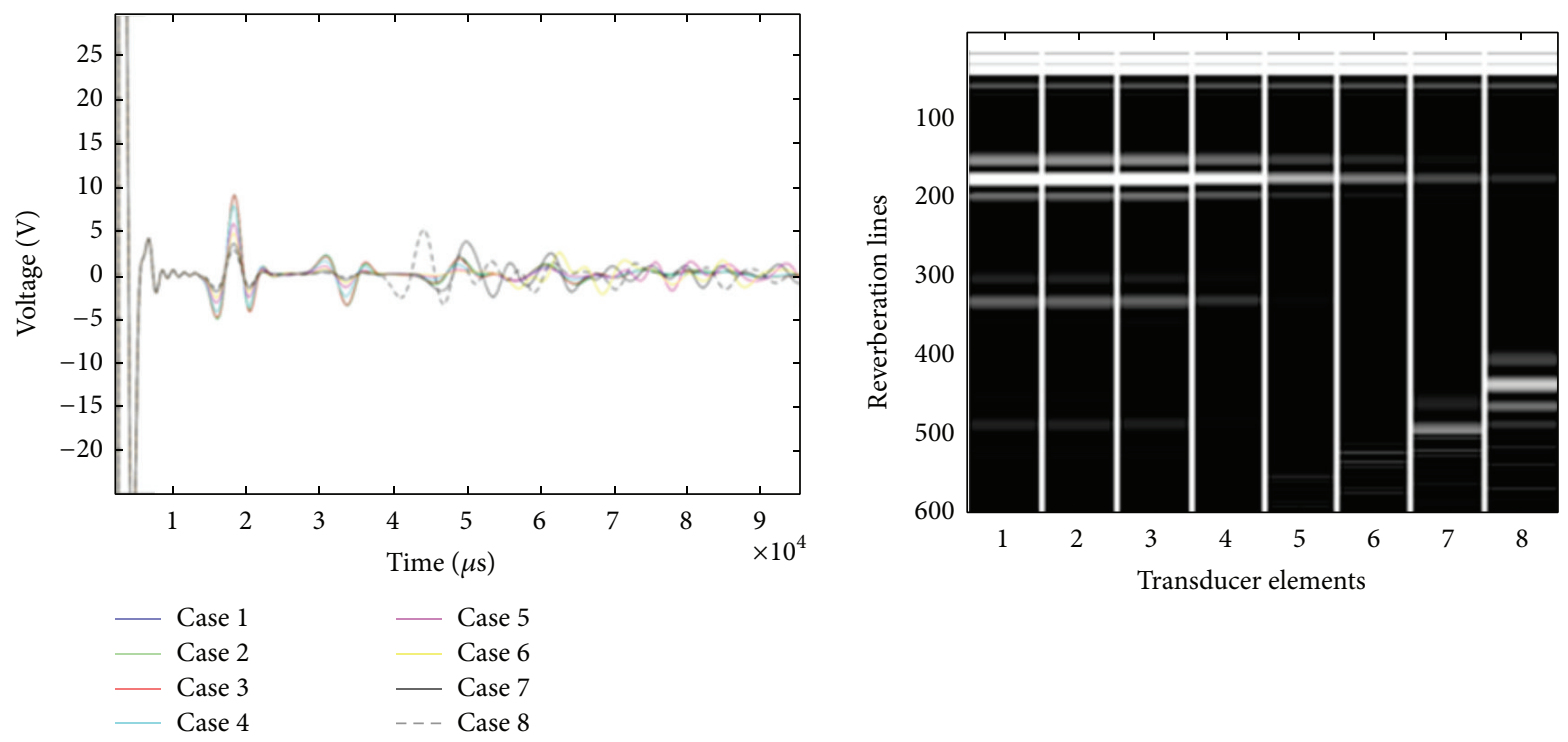

(d)

FIGURE 6: Voltage amplitudes responses (left) and the corresponding in-air reverberation patterns (right) for various simulated damage to the following transducer layers: (a) backing layer; (b) matching layer; (c) crystal layer. (d) Lens layer. In-air reverberation patterns are given for eight damage severities indicated by integer numbers, where 1 (left image) corresponds to the full breakage and 8 (right) corresponds to the smallest damage severity, as indicated in Table 4.

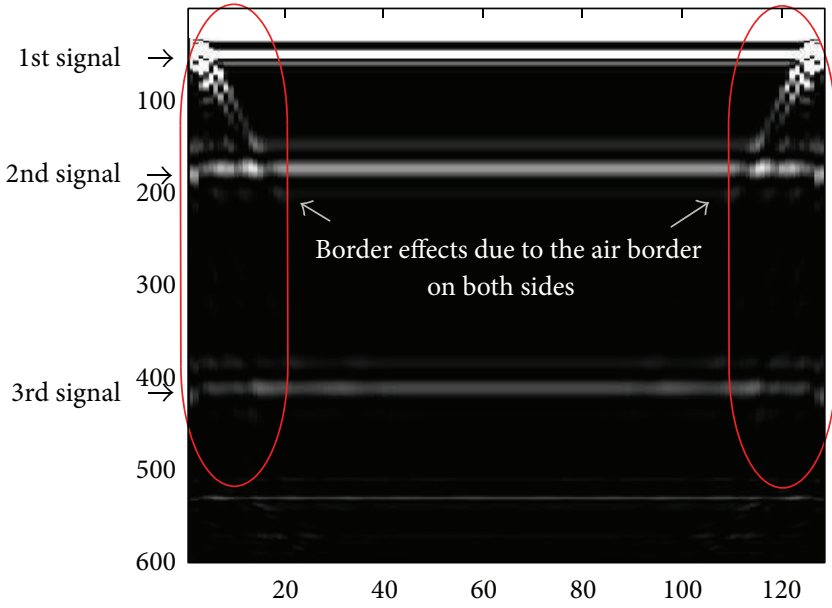

FIGURE 7: In-air reverberation pattern for the 128-finger healthy medical ultrasonic transducer (the vertical numbers corresponded to the reverberations line and the horizontal numbers correspond to the number of elements). Signals 1, 2, and 3 on the right hand side correspond to the signal paths and reflections indicated in Figure 4.

is similar to the effect observed for the damaged backing layer in Figure 8(a). However, it is more pronounced and can be seen through the entire depth of the image. This has been already observed when the one-element transducer was analyzed in Figure 6(c). The ultrasonic waves are not able to leave the crystals in the broken fingers and do not travel in upwards or downwards directions. It is important to note that in a clinical image or a reverberation image, ceramic damage normally produces a dark region in the image. This region appears below the damaged fingers and is dark in appearance because the crystals are transmitting less acoustic energy and therefore receiving less acoustic energy. However, damage in the current investigations was modelled using stiffness reduction. Future developments will focus on more realistic models that involve modifications to piezoelectric coefficients.

Figure 8(d) shows the reverberation image for a transducer with a lens layer that is damaged over a width of $3.4 \mathrm{~mm}$ in the middle of the transducer. In the top part the reverberation pattern displays an image that is similar to the healthy transducer in Figure 7. However, a few bright lines further down the image can be observed in the damaged area of the transducer due to the reflection at the damaged lens layer. The high reflection coefficient at this border reflects the wave completely and no more reflections can be seen when the pattern is analyzed towards the bottom; the area is dark. A similar effect was observed for the one-finger transducer in Figure 6(d).

\section{Conclusions}

A finite element model was used to simulate the in-air reverberation pattern generated by medical ultrasonic array transducers. Furthermore numerical simulations were used to demonstrate changes in the simulated in-air reverberation pattern when electromechanical changes were introduced to the backing matching, crystal, and lens layers of the transducer.

The results of numerical simulations demonstrate that pulse propagation modelling, within layers of a simplified medical ultrasonic transducer, produces reverberation pattern 


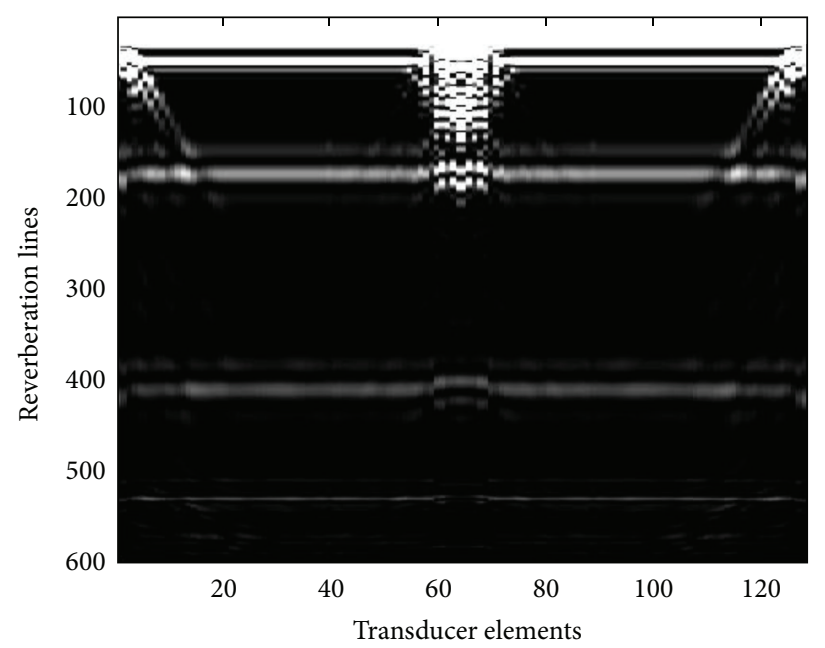

(a)

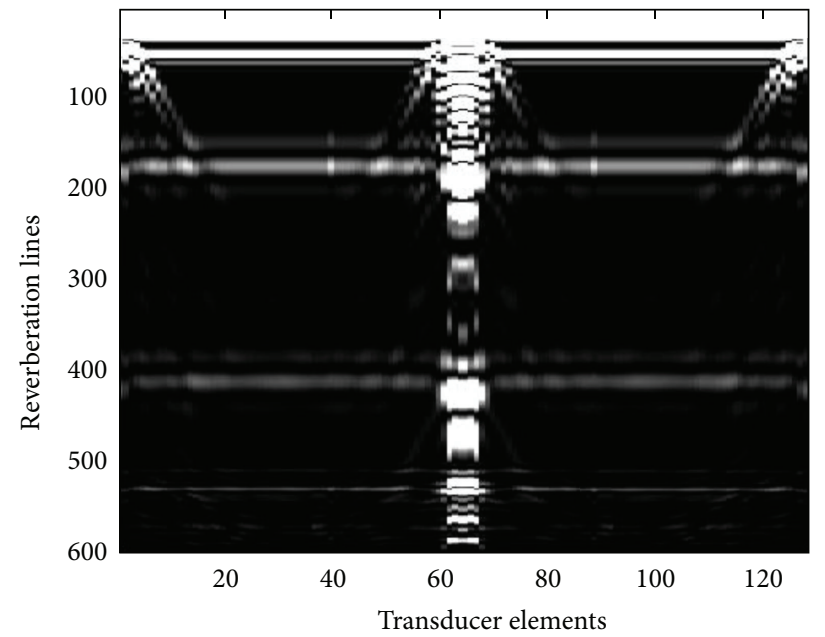

(c)

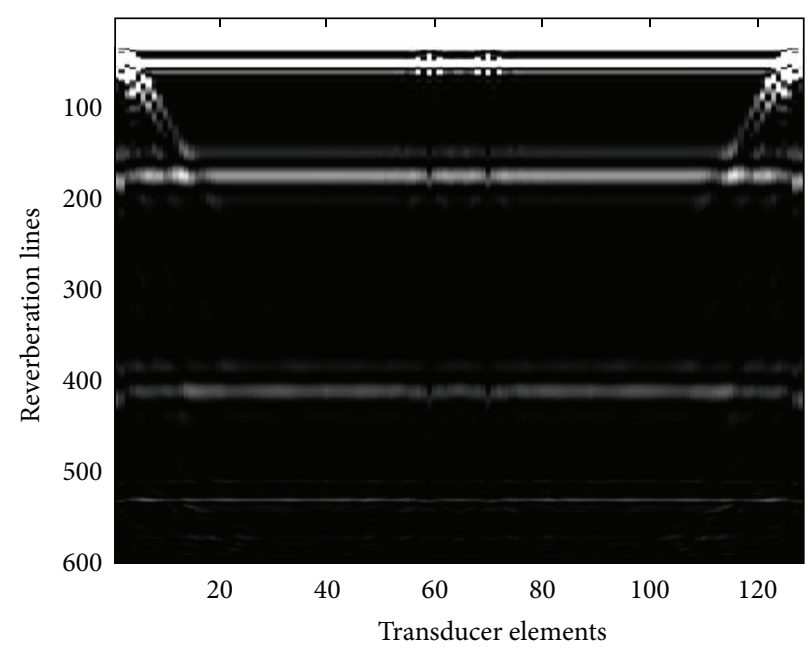

(b)

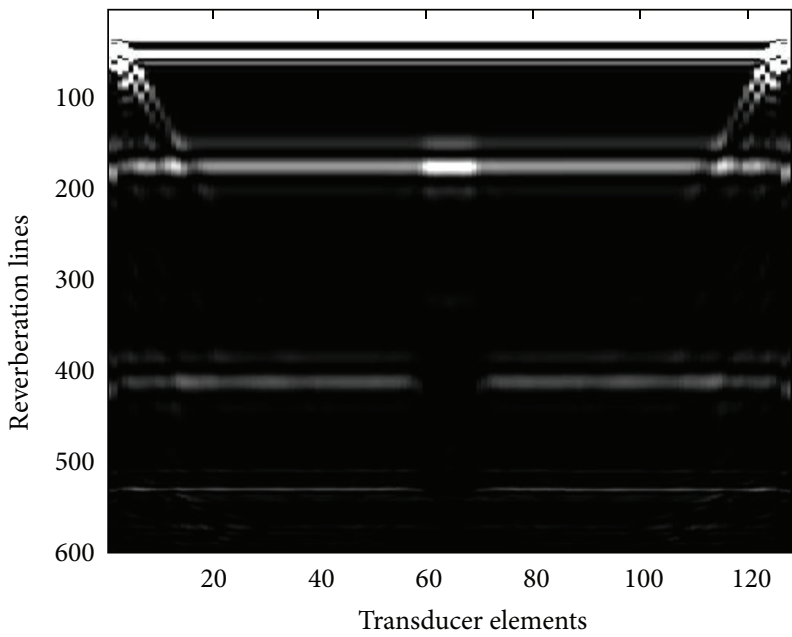

(d)

FIGURE 8: In-air reverberation patterns for the 128-element medical ultrasonic transducer for the following damaged elements: (a) backing layer; (b) matching layer; (c) crystal; and (d) lens.

images that are similar to those seen experimentally. Analysis of the simulated results reveals that damage to crystal layer has the largest effect on the simulated in-air reverberation pattern. The next worst case is damage to the lens layer. Damage to the backing layer is the most difficult one to detect for small reductions of Young's modulus. The simulated results show that in-air reverberation patterns can uniquely reveal faults to the different layers of a transducer. This exploratory approach may help to provide a better explanation of transducer faults and also help with the earlier detection of transducer faults.

It is anticipated that these encouraging results will generate further studies that correlate subtle changes in in-air reverberation images with real faults in medical ultrasonic transducers. Future work should focus on more accurate transducer and fault modelling that is validated by experimental results from damaged and healthy medical transducers. Other future works will look to extend the modelling into a full 3D geometry so that more realistic faults can be investigated. Modelling of pulse transmission and detection will also incorporate multiple cycle acoustic pulses such as those used in pulse wave and colour Doppler imaging.

\section{Conflict of Interests}

The authors declare that there is no conflict of interests regarding the publication of this paper.

\section{Acknowledgments}

All authors from the AGH University of Science and Technology would like to acknowledge the support of the Foundation for Polish Science under the research project WELCOME no. 2010-3/2 (Innovative Economy, National Cohesion Programme, and EU). 


\section{References}

[1] M. Mårtensson, M. Olsson, B. Segall, A. G. Fraser, R. Winter, and L.-Å. Brodin, "High incidence of defective ultrasound transducers in use in routine clinical practice," European Journal of Echocardiography, vol. 10, no. 3, pp. 389-394, 2009.

[2] M. Mårtensson, M. Olsson, and L.-Å. Brodin, "Ultrasound transducer function: annual testing is not sufficient," European Journal of Echocardiography, vol. 11, no. 9, pp. 801-805, 2010.

[3] S. D. Pye, W. Ellis, and T. MacGillivray, "Medical ultrasound: a new metric of performance for grey-scale imaging," Journal of Physics: Conference Series, vol. 1, pp. 187-192, 2004.

[4] N. M. Gibson, N. J. Dudley, and K. Griffith, "A computerised quality control testing system for B-mode ultrasound," Ultrasound in Medicine and Biology, vol. 27, no. 12, pp. 1697-1711, 2001.

[5] M. M. Goodsitt, P. L. Carson, S. Witt, D. L. Hykes, and J. M. Kofler, "Real-time B-mode ultrasound quality control test procedures: report of AAPM Ultrasound Task Group No. 1," Medical Physics, vol. 25, no. 8, pp. 1385-1406, 1998.

[6] N. J. Dudley, K. Griffith, G. Houldsworth, M. Holloway, and M. A. Dunn, "A review of two alternative ultrasound quality assurance programmes," European Journal of Ultrasound, vol. 12, no. 3, pp. 233-245, 2001.

[7] R. Price, Report No 70. Routine Quality Assurance of Ultrasound Imaging Systems, Institute of Physics and Engineering in Medicine (IPEM), York, UK, 1995.

[8] S. Russell, "Quality assurance of ultrasound imaging systems," Tech. Rep. No. 102, Institute of Physics and Engineering in Medicine (IPEM), York, UK, 2010.

[9] T. Quinn and P. K. Verma, "The analysis of in-air reverberation patterns from medical ultrasound transducers," Ultrasound, vol. 22, no. 1, pp. 26-36, 2014.

[10] W. P. Mason, Electromechanical Transducers and Wave Filters, Van Nostrand, New York, NY, USA, 2nd edition, 1942.

[11] C. Dang, L. W. Schmerr Jr., and A. Sedov, "Modeling and measuring all the elements of an ultrasonic nondestructive evaluation system I: modeling foundations," Research in Nondestructive Evaluation, vol. 14, no. 3, pp. 141-176, 2002.

[12] M. Redwood, "Transient performance of a piezoelectric transducer," Journal of the Acoustical Society of America, vol. 33, no. 4, pp. 527-536, 1961.

[13] R. Krimholtz, D. A. Leedom, and G. L. Matthaei, "New equivalent circuits for elementary pizoelectric transduces," Electronics Letters, vol. 6, no. 13, pp. 398-399, 1970.

[14] G. Caliano, A. Caronti, M. Baruzzi et al., "PSpice modeling of capacitive microfabricated ultrasonic transducers," Ultrasonics, vol. 40, no. 1-8, pp. 449-455, 2002.

[15] L. Wu and Y. C. Chen, "PSPICE approach for designing the ultrasonic piezoelectric transducer for medical diagnostic applications," Sensors and Actuators A: Physical, vol. 75, no. 2, pp. 186-198, 1999.

[16] B. Fu, C. Li, J. Zhang, and Z. Huang, "Modelling of piezoelectric Langevin transducers by using mixed transfer matrix methods," Journal of the Korean Physical Society, vol. 57, no. 4, pp. 929-932, 2010.

[17] E. K. Rahani and T. Kundu, "Gaussian-DPSM (G-DPSM) and element source method (ESM) modifications to DPSM for ultrasonic field modelling," Ultrasonics, vol. 51, no. 5, pp. 625631, 2011.

[18] R. Marklein, "The finite integration technique as a general tool to compute acoustic, electromagnetic, elastodynamic, and coupled wave fields," in Review of Radio Science: 1999-2002 URSI, W. R. Stone, Ed., pp. 201-244, IEEE Press, Piscataway, NJ, USA, John Wiley \& Sons, New York, NY, USA, 2002.

[19] M. Spies, "Modeling of transducer fields in inhomogeneous anisotropic materials using Gaussian beam superposition," NDT \& E International, vol. 33, no. 3, pp. 155-162, 2000.

[20] M. M. Rahman and S. Chowdhury, "Square diaphragm CMUT capacitance calculation using a new deflection shape function," Journal of Sensors, vol. 2011, Article ID 581910, 12 pages, 2011.

[21] D. Engelke, B. Oehme, and J. Strackeljan, "A novel drive option for piezoelectric ultrasonic transducers," Modelling and Simulation in Engineering, vol. 2011, Article ID 910876, 6 pages, 2011.

[22] R. Lerch, "Simulation of piezoelectric devices by two- and threedimensional finite elements," IEEE Transactions on Ultrasonics, Ferroelectrics, and Frequency Control, vol. 37, no. 3, pp. 233-247, 1990.

[23] A. Iula, F. Vazquez, M. Pappalardo, and J. A. Gallego, "Finite element three-dimensional analysis of the vibrational behaviour of the Langevin-type transducer," Ultrasonics, vol. 40, no. 1-8, pp. 513-517, 2002.

[24] N. N. Abboud, G. L. Wojcik, D. K. Vaughan, J. Mould, D. J. Powell, and L. Nikodym, "Finite element modeling for ultrasonic transducers," in Medical Imaging: Ultrasonic Transducer Engineering, vol. 3341 of Proceedings of SPIE, pp. 19-42, San Diego, Calif, USA, February 1998.

[25] M. Greenspan, "Piston radiator: some extensions of the theory," Journal of the Acoustical Society of America, vol. 65, no. 3, pp. 608-621, 1979.

[26] B. A. Auld, Acoustic Fields and Waves in Solids, vol. 1-2, Krieger Publishing, 2nd edition, 1990.

[27] E. Filoux, S. Callé, D. Certon, M. Lethiecq, and F. Levassort, "Modeling of piezoelectric transducers with combined pseudospectral and finite-difference methods," Journal of the Acoustical Society of America, vol. 123, no. 6, pp. 4165-4173, 2008.

[28] E. F. Levassort, S. Callé, D. Certon, M. Lethiecq, and E. Filoux, "Single-element ultrasonic transducer modeling using a hybrid FD-PSTD method," Ultrasonics, vol. 49, no. 8, pp. 611-614, 2009.

[29] F. Schubert and B. Lamek, "3-D ultrasonic transducer modeling using the elastodynamic finite integration technique in combination with point-source-synthesis," in Proceedings of the 4th International Workshop-NDT in Progress, pp. 1-10, Prague, Czech Republic, November 2007.

[30] T. Kundu, D. Placko, E. K. Rahani, T. Yanagita, and C. M. Dao, "Ultrasonic field modeling: a comparison of analytical, semianalytical, and numerical techniques," IEEE Transactions on Ultrasonics, Ferroelectrics, and Frequency Control, vol. 57, no. 12, pp. 2795-2807, 2010.

[31] ANSYS, http://www.ansys.com/.

[32] J. Kocbach, Finite element modeling of ultrasonic piezoelectric transducers [Ph.D. thesis], Department of Physics, University of Bergen, 2000.

[33] S. Metcalfe, S. Iball, T. Evans et al., "Reproducibility of quality assurance test parameters-a multi user trial," in Proceedings of the British Medical Ultrasound Society Annual Conference, Manchester, UK, 2005.

[34] A. Khalatkar, V. K. Gupta, and A. Agrawal, "Analytical, FEA, and experimental comparisons of piezoelectric energy harvesting using engine vibrations," Smart Materials Research, vol. 2014, Article ID 741280, 8 pages, 2014. 
[35] L. Malgaca and H. Karagülle, "Numerical and experimental study on integration of control actions into the finite element solutions in smart structures," Shock and Vibration, vol. 16, no. 4, pp. 401-415, 2009.

[36] A. Nechibvute, A. Chawanda, and P. Luhanga, "Finite element modeling of a piezoelectric composite beam and comparative performance study of piezoelectric materials for voltage generation," ISRN Materials Science, vol. 2012, Article ID 921361, 11 pages, 2012.

[37] W. M. Rubio, F. Buiochi, J. C. Adamowski, and E. C. N. Silva, "Modeling of functionally graded piezoelectric ultrasonic transducers," Ultrasonics, vol. 49, no. 4-5, pp. 484-494, 2009.

[38] M. Boeff, Finite Element Analysis of an Ultrasonic Transducer, Department of Mechanical Engineering, University of Sheffield, Sheffield, UK, 2010.

[39] W. R. Hendrick, D. L. Hykes, and D. E. Starchman, Ultrasound Physics and Instrumentation, Elsevier, St. Louis, Mo, USA, 2005. 

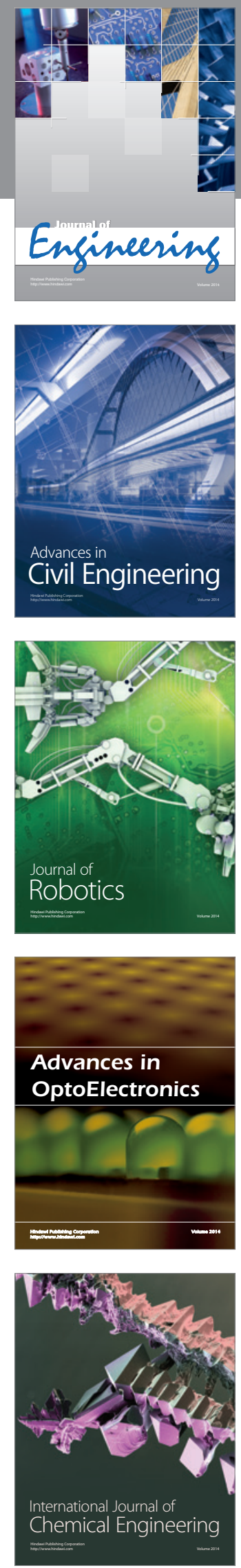

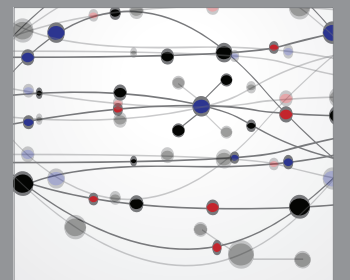

The Scientific World Journal
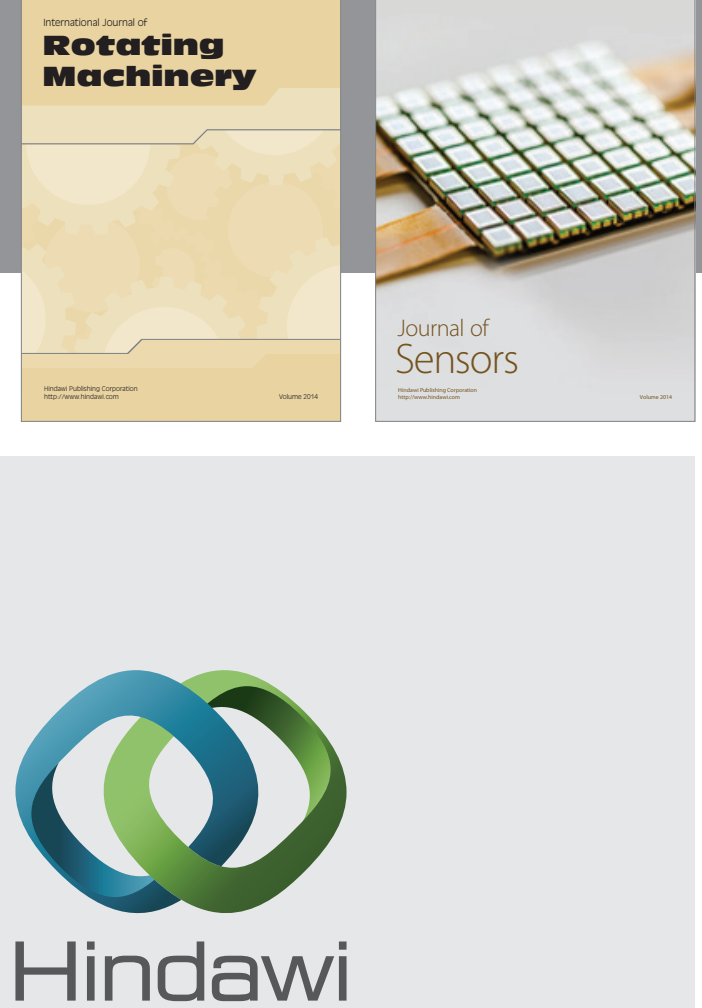

Submit your manuscripts at http://www.hindawi.com
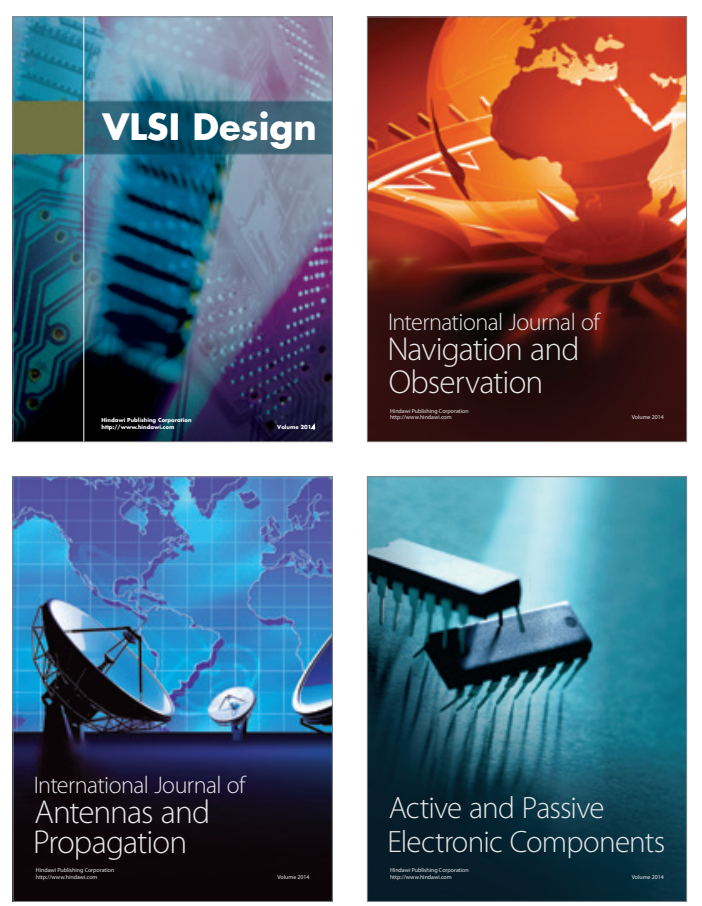
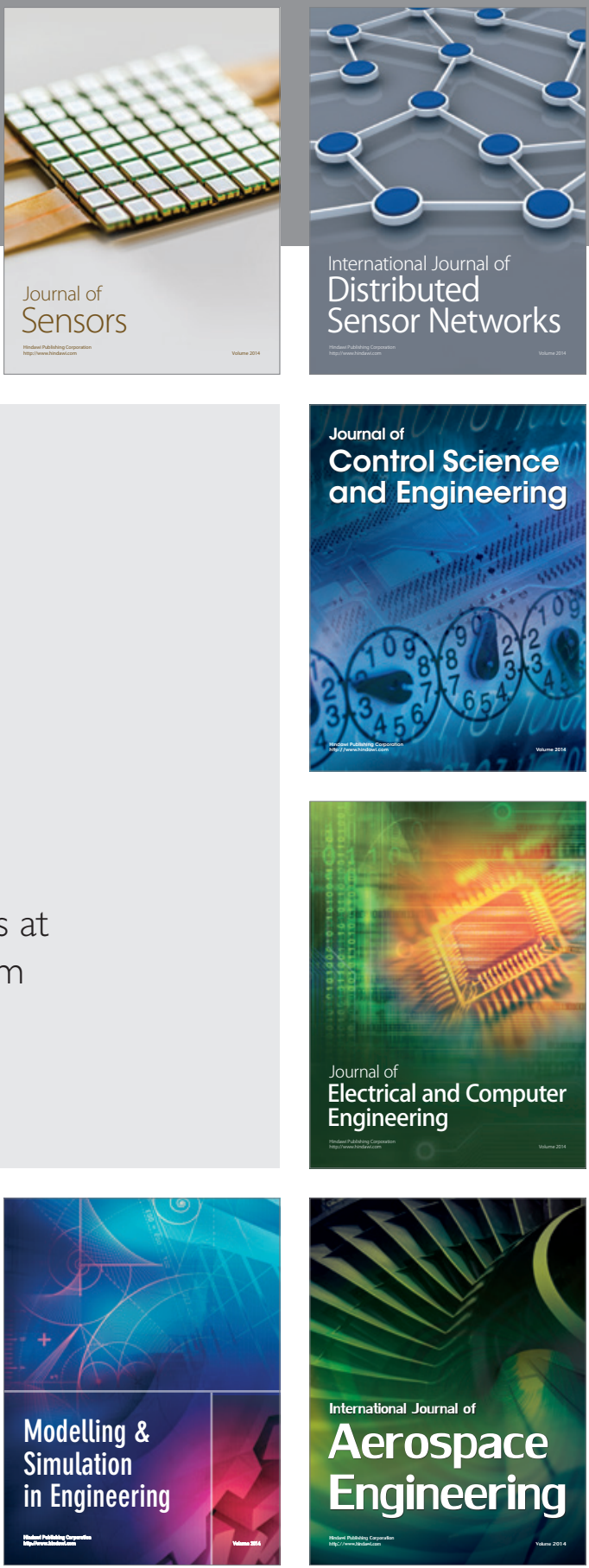

Journal of

Control Science

and Engineering
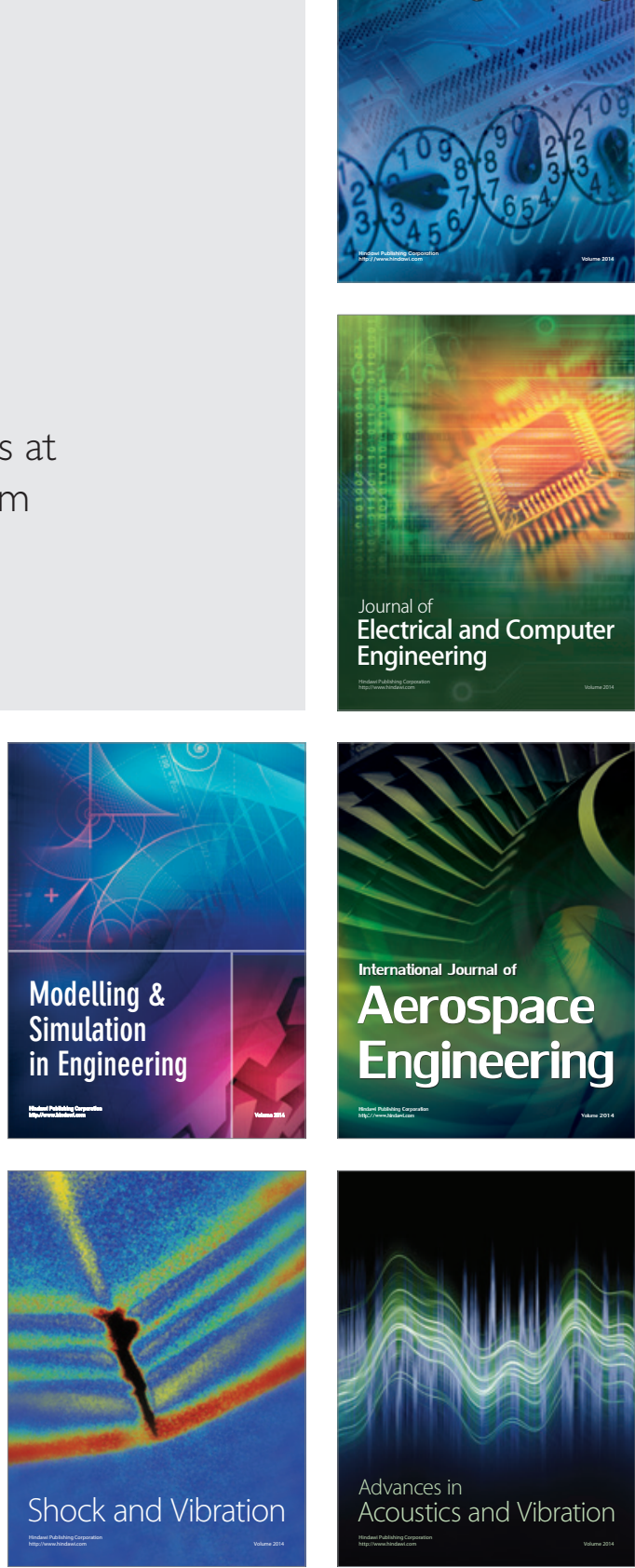